\title{
Spatially resolved determination of toxic trace elements in plants of Panasqueira mining region using micro $\mathrm{X}$-ray fluorescence
}

M. Guerra*, S. C. Pé-Leve Santos ${ }^{*}$, A. M. E. Barroso*, C. P. S. Fonseca*, M. Eloy Cruz*, P. Amaro , J. L. Figueirinhas $^{* * * * *,}$, M. L. Carvalho*, J. P. Santos ${ }^{*}$

* Centro de Física Atómica (CFA), Departamento de Física, Faculdade de Ciências e Tecnologia (FCT), Universidade Nova de Lisboa, 2829-516 Monte da Caparica, Portugal

** CFMC-UL, Av. Prof. Gama Pinto 2, 1649-003 Lisboa, Portugal

**** Departamento de Física, IST-UTL, Av. Rovisco Pais 1, 1049-001 Lisboa, Portugal

\section{Email: mguerra@fct.unl.pt}

Mining activities have an important environmental impact because of the interaction of extracted metals with the different elements of the environment, namely the vegetation around the mining site [1]. In the Panasqueira mine, located in Beira Baixa, near the town of Barroca Grande, in central Portugal, elements such as tungsten, iron, copper and tin were extracted over the years, and consequently were being deposited in the surface, creating environmental hazards not only in the soils but also in plants and especially in the underground water reserves.

To our knowledge, this is the first report of the in situ distribution and speciation of $\mathrm{As}, \mathrm{Cu}, \mathrm{Zn}$ and $\mathrm{W}$, in hydrated (and fresh) plant tissues, providing valuable information on the potential mechanisms by which they are toxic. These mechanisms remain unclear, despite their toxic effects having been researched for more than 100 years. Furthermore, the mechanisms used by plants to tolerate excess trace metals continue to be debated. To shed light on these issues, it is important, if not indispensable, to determine the distribution of trace metals within the root tissue. For this purpose, we contend that it is necessary to investigate the spatially resolved distribution and speciation of such elements using hydrated (and preferably, fresh) plant tissues collected near contaminated sites [2].

To analyze the plants collected in Panasqueira mining area, we have used two apparatus: the energy dispersive X-ray Fluorescence (EDXRF) spectrometer equipped with a commercial X-ray tube (PW1140, $100 \mathrm{kv}, 80 \mathrm{~mA}$ ) in a triaxial geometry and the Bruker's Tornado micro X-ray fluorescence (MXRF) spectrometer.

Samples from various plant species, namely Rubus sp., Pinus pinaster, Rosa sp., Retama sphaerocarpa, Pteridium aquilinum, Erica sp., Arbutus unedo and Acacia dealbata, were collected and placed in identified plastic bags. These samples were collected in the mine's new wash site, in Barroca Grande, and also in the old wash site, labeled as Rio. For control, the same species were collected in Silvares, about $4 \mathrm{~km}$ to the east of the wash sites. The elemental concentrations measured with the EDXRF spectrometer are displayed in Fig. (1). We can see that $\mathrm{W}$ is observed in the contaminated samples, mainly in Barroca Grande). Regarding other toxic elements such as $\mathrm{As}, \mathrm{Br}$, and $\mathrm{Rb}$, we see a clear trend in the species collected near the mine wash sites, which indicates much higher concentrations of these elements when compared to the plants in the control region. We emphasize that in every sample collected in this study, we have found much higher values of As than those imposed by the World Health Organization (WHO) even in the control samples, which warrants further investigation.

Assorted collected plants, and respective fruits, were also studied using the MXRF spectrometer, namely the Arbutus unedo. This plant's fruit is used to make an alcoholic drink very popular in the region. In Fig. (2) are displayed images of the Arbutus unedo fruit, collected in the contaminated zone, cut in half, that the 
elemental spatial distribution. We observe that the $\mathrm{W}$ and As are homogeneously distributed, and their concentrations are as high as in the plants analyzed with the EDXRF spectrometer, and higher than the imposed by the WHO. On the other hand, we see that iron and potassium tend to be present at concentrations that are in accordance with the WHO standards (although near the high end) and they are mainly distributed in the outermost sections of the plants, such as the fruit shell.

[1] S. C. Pé-Leve Santos, et al; J. Soils and Sed. 14, 778-784, 2014.

[2] O. Gonzalez-Fernandez, et al; X-Ray Spectrom, 40, 353-363, 2011.

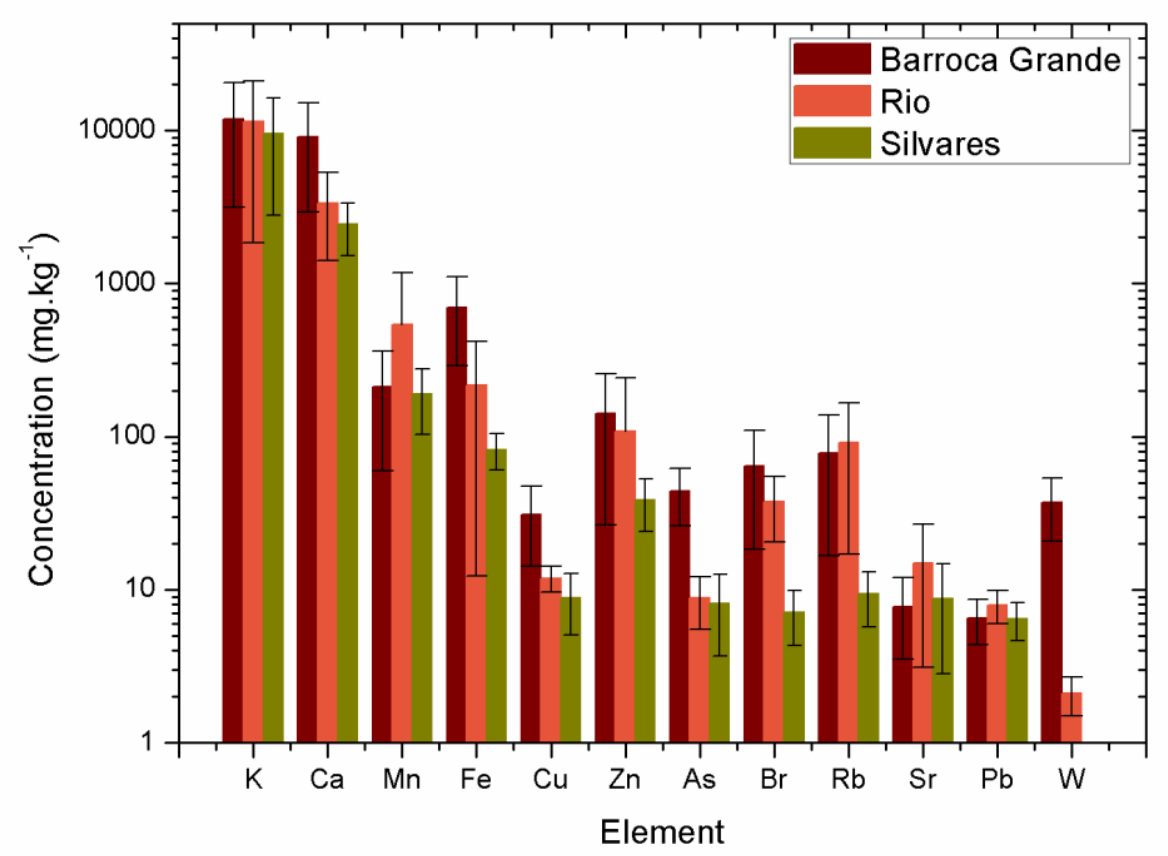

Figure 1. Comparison of average elemental concentration (milligram per kilogram) between all collected species near the regions of Barroca Grande (current wash site), Rio (old wash site), and Silvares (control). The error bars represent the statistical standard deviation.

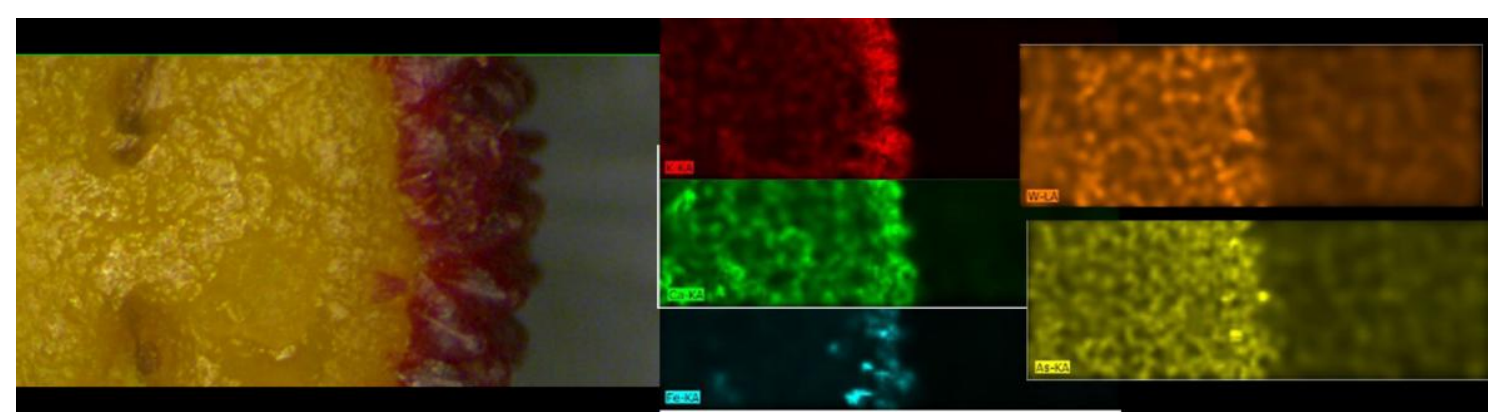

Figure 2. The leftmost image shows an Arbutus unedo fruit cut in half. The five images on the right show the concentrations of the respective court. The red, green, blue, orange and yellow images show the spatially resolved distribution of $\mathrm{K}, \mathrm{Ca}, \mathrm{Fe}, \mathrm{W}$ and $\mathrm{As}$, respectively. 